\title{
Antibiotics without Prescriptions: US \& Indian Perspective
}

\author{
Nancy Khardori ${ }^{1} \cdot$ Chand Wattal $^{2}$
}

Received: 5 November 2019 / Accepted: 5 November 2019 / Published online: 6 December 2019

(C) Dr. K C Chaudhuri Foundation 2019

NK- There has been (and still is) overwhelming and redundant published material on the gravity of the public health problem that resistance to antimicrobial agents poses. Often, the reviews of the topics convey the impression that such resistance is emerging and unexpected, and therefore, there is a need to create pathways to curb it. The editorial in a recent issue of Indian Journal of Medical Research entitled "Creating political commitment for antimicrobial resistance in developing countries" reviews how the world bodies, including the World Health Organization, have been advocating their member states to develop national action plans to address appropriate antimicrobial use [1].

$\mathrm{CW}$ - Antibiotic resistance has become a major public health problem world over, and all efforts are needed to slow its emergence. A pilot study was conducted in New Delhi, India, for establishing a methodology for surveillance of antimicrobial use and resistance in resourceconstrained settings which showed high levels of antibiotic use and resistance in the community [2, 3]. In its second phase, a qualitative study was conducted to ascertain stakeholders like doctors', pharmacists' and high school children's behavior relating to antibiotic use and the factors influencing prescribing patterns among primary care physicians [4]. Various qualitative studies have looked at the prescribing behavior of general practitioners (GPs) in the Western world [5, 6].

CW - Chand Wattal

NK - Nancy Khardori

Nancy Khardori

nkhardori@gmail.com

Chand Wattal

chandwattal@gmail.com

1 Infectious Diseases, Solid Organ Transplant Program at Sentara Norfolk General Hospital, Norfolk, Virginia, USA

2 Clinical Microbiology \& Immunology, Sir Ganga Ram Hospital, Rajinder Nagar, New Delhi, India
$\mathrm{CW}$ - Important factors identified as drivers of inappropriate antibiotic prescribing include patient pressure [7], desire to preserve the doctor-patient relationship, the doctor's personal characteristics [8], uncertainty about which patients will benefit from antibiotics, the daily pressures of clinical general practice [9], the patient's presenting complaints and findings on physical examination [10]. Three broad themes identified were: behavioral characteristics of doctors and patients; laxity in regulation of prescribing and dispensing antibiotics and intervention strategies to decrease misuse of, and resistance to, antibiotics.

NK- The fact that antimicrobial resistance will develop was actually known at the same time as the very first antibiotic (penicillin) became available for public consumption in 1944. The discoverer of penicillin, Alexander Fleming (who has been called the accidental hero of medicine), said in New York Times June 26, 1945 [11].

"The greatest possibility of evil in self-medication is the use of too small doses so that instead of cleaning up infection the microbes are educated to resist penicillin and a host of penicillin-fast organisms is bred out which can be passed to other individuals and from them to others until they reach someone who gets a septicemia or pneumonia which penicillin cannot save."

In this quote, Fleming was merely scratching the surface of what was to become of antimicrobial agent use. However, this quote attests to the significance of learning the fundamental concepts of interaction between antimicrobial agents and microbes, which is something no policymakers or world bodies can teach the medical profession.

To quote Frank L Meleny, a practicing surgeon from 1947 [12].

"We have to get experience all over again on the behavior of infection under treatment with these new drugs. There is a temptation to use them promiscuously and yet 
certainly if we are to improve our results we must use them with discrimination."

When one adds to this lack of emphasis on learning the "ABCs" of antimicrobial use, the crossover (e.g., extensive use of antibacterial agents for infections caused by viruses), selection of antibacterial agents based on preferences and business dealings, and inattention to and/or lack of ability to interpret microbiology laboratory results, one cannot justify the surprise expressed in writings on antimicrobial resistance. The icing on the cake that feeds the very bacteria we wish to destroy is the distribution of these agents without the patient being seen by a medical provider, solely based on the choice of "drug dispensers" and users themselves. When I accepted the invitation to participate in writing for and editing this dedicated issue of the Indian Journal of Pediatrics, my intention was to focus on the non-prescription availability of antimicrobial agents in many parts of the world including India.

CW- The serious issue here in India is not only the inappropriate prescriptions but no prescriptions at all. Lack of implementation of rules and regulations like schedule $\mathrm{H} 1$ which allows such practices get rampant. Health being a state subject creates further conflicts in implementing centre recommended regulations. The enforcement agencies have their share of limitations by way of trained human resources. There could be only one Drug inspector in whole of the state with a few to assist. Lack of knowledge in masses complicates the matter further. More often than not, antibiotics are viewed as strong medicines to get alright overnight to peruse ones vocation more so if it is the sole earning member of the family. The very knowledge of most of upper respiratory tract infection (URTI) and diarrhea proves that they are viral in nature and self-limiting and antibiotics have no role either. The medical community also needs to work in the community for their awareness. An attempt has been made by the government to indicate to the public by using a red line over the packs of medicines containing antibiotics so that the consumer gets aware that he or she has been prescribed an antibiotic or has gone to purchase medicines without prescription with the help of the pharmacist. Public at large need to be informed about the collateral damages that such self-medications cause. The common concept of resistance is viewed as the human being gets resistant when it is the microorganism that is ragging the patient and is developing resistance. The prescribers also add to the problem by providing consultations over the phone or other modes of communication without having examined the patient. This amounts to an activity similar to consumption of antibiotics without a prescription.

NK- I have been learning, teaching and practicing medicine in the United States since 1977 where all antibiotic prescriptions are provided by health care providers. That being so, should we have any especially significant antibiotic resistance here? Since we know that is not the case, it is clear that health care providers with authority to prescribe antibiotics are contributing no less to the ongoing development of resistance in the microbes than the unauthorized drug dispensers and selfprescribers. Antimicrobial agents by definition are selectively toxic to microbes and therefore are relatively safe for the human tissues. One can say that this safety profile (as contrasted, for example, with cancer chemotherapeutic agents with nonselective toxicity against replicating cells) is responsible at the core for "fearless" and "thoughtless" prescriptions and nonprescription use of antibiotics. In short, a scientific miracle that is effective as well as relatively safe for human and animal use has been turned into a disaster where we now must reckon with microbes that cannot be treated by any of the available antibiotics, Simultaneously, the patient population that is highly susceptible to usual and unusual microbial infections have expanded (through other medical miracles).

NK- This realization takes us back to the ABCs of medical education. A few years ago, I participated in a national medical conference in India. I heard, a very prominent senior medical educator share with a large audience that "In India we do not need to develop infectious diseases as a specialty of medicine since our students in their fourth year of medical school have been given knowledge base and are equipped with treating infectious diseases." I am unprepared to hear it at a national conference dedicated to the appropriate management of infectious diseases that remain the number one cause of death in India. The health care system in the country has matured into providing advanced and expensive treatments such as cancer care and organ transplantation, in addition to becoming an attractive destination for medical tourism. The management of antimicrobial resistance in all parts of the world needs to start with the ABCs of medical education, go to the middle alphabet letters during graduate and postgraduate training, and end up with the XYZs, the latter being policies encompassing public awareness and education as well as restriction of drug prescribing-including antimicrobial agents-to educated and trained health care providers.

CW- We need to preserve the extremely precious resource in the name of "antibiotics" for the future generations to come and need to use it only when required and with a valid prescription from an authorised practitioner of modern medicine. Quacks have further messed the situation with impunity and go around their business prescribing antibiotics without any training or knowledge and such prescriptions are also equivalent to taking antibiotics without a valid prescription.

$\mathrm{CW}$ - Further to this non-human use of high end antibiotics like colistin is a cause to worry.

It is high time that the medical fraternity work with the enforcement agencies and policy makers to stop the menace of use of antibiotics without a valid prescription and stops its availability across the counter.

NK-Finally let us understand that encouraging pharmaceutical industry to make new antimicrobial agents should be 
viewed as a bonus rather than the answer to the current crisis. We must not ignore the fact that all living beings including microbes are pursuing the fundamental of survival against known and new threats. The persistence of microbes that have been rendered resistant and untreatable by overuse, misuse and abuse of antibiotics is only proving the Darwinian principle of survival of the fittest.

\section{Compliance with Ethical Standards}

Conflict of Interest None.

\section{References}

1. Bhatia R, Katoch YM, Inoue H. Creating political commitment for antimicrobial resistance in developing countries. Indian J Med Res. 2019;149:83-6.

2. Kotwani A, Holloway K, Chaudhury RR. Methodology for surveillance of antimicrobials use among out-patient in Delhi. Indian J Med Res. 2009;129:555-60.

3. Wattal C, Raveendran R, Kotwani A, et al. Establishing a new methodology for monitoring of antimicrobial resistance and use in the community in a resource poor setting. J Appl Ther Res. 2009;7: $16-24$.

4. Kotwani A, Wattal C, Katewa S, Joshi PC, Holloway K. Factors influencing primary care physicians to prescribe antibiotics in Delhi, India? Fam Pract. 2010;27:684-90.
5. Crowe S, Tully MP, Cantrill JA. The prescribing of specialist medicines: what factors influence GPs' decision making? Fam Pract. 2009;26:301-8.

6. Wood F, Simpson S, Butler CC. Socially responsible antibiotics choices in primary care: a qualitative study of GP's decisions to prescribe broad-spectrum and fluoroquinolone antibiotics. Fam Pract. 2007;24:427-34.

7. Hart AM, Pepper GA, Gonzales R. Balancing acts: deciding for or against antibiotics in acute respiratory infections. J Fam Pract. 2006;55:320-5.

8. Butler CC, Rollnick S, Pill R, Maggs-Rapport F, Stott N. Understanding the culture of prescribing: a qualitative study of general practitioners' and patients' perceptions of antibiotics for sore throats. BMJ. 1998;317:637-42.

9. Kumar S, Little P, Britten N. Why do general practitioners prescribe antibiotics for sore throat? Grounded theory interview study. BMJ. 2003;326:138-43.

10. Fischer T, Fischer S, Kochen MM, Hummers-Pradier E. Influence of patient symptoms and physical findings on general practitioners' treatment of respiratory tract infections: a direct observational study. BMC Fam Pract. 2005;6:6.

11. Penicillin's Finder Assays its Future. New York Times. June 26, 1945: 21.

12. Meleney FL. Treatment of surgical infections by chemical and antibiotic agents. Postgrad Med. 1947;1:87-96.

Publisher's Note Springer Nature remains neutral with regard to jurisdictional claims in published maps and institutional affiliations. 